\title{
Albumin excretion in diabetic patients in the setting of acute myocardial infarction: association with 3-year mortality
}

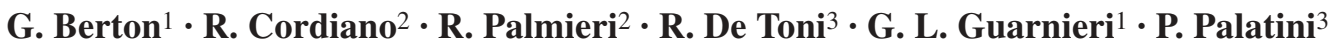 \\ ${ }^{1}$ Department of Internal Medicine and Cardiology, Conegliano General Hospital, Conegliano, Italy \\ ${ }^{2}$ Department of Internal Medicine and Cardiology, Adria General Hospital, Adria, Italy \\ ${ }^{3}$ Department of Clinical and Experimental Medicine, University of Padova, Clinica Medica, Padova, Italy
}

\section{Abstract}

Aims/hypothesis. Diabetes mellitus is associated with increased mortality in subjects with acute myocardial infarction (AMI). We aimed to estimate the risk of mortality in AMI patients with and without diabetes using the urinary albumin : creatinine ratio (ACR).

Methods. This is a prospective study of 121 consecutive, non-selected diabetic AMI patients, 121 age- and sex-matched non-diabetic AMI patients and 61 diabetic non-AMI outpatients as control subjects. All data were obtained during the first 7 days of hospitalisation and each AMI patient was followed for a period of exactly 3 years. Baseline ACR RIA measurements were made on the 1 st, 3 rd and 7 th days of admission.

Results. Adjusted ACR values were significantly higher in the diabetic AMI patients than in the diabetic control outpatients $(p<0.0001)$, and a significant difference was observed between the weekly ACR slopes for these two groups $(p<0.0001)$. Microalbuminuria was more prevalent in the diabetic AMI patients than in the non-diabetic AMI patients on the 1st day $(62 \%$ vs $46 \%, p=0.01)$ and 3 rd day $(41 \%$ vs $29 \%$, $p=0.04)$. Among the AMI patients with normoalbuminuria (ACR $<30 \mu \mathrm{g} / \mathrm{mg}$ ), the mortality rate was $11.6 \%$ for the patients without diabetes and $33.8 \%$ for those with diabetes $(p=0.001)$. The mortality rate was much higher among the AMI patients with microalbuminuria (ACR $\geq 30 \mu \mathrm{g} / \mathrm{mg}$ ) and similar for the diabetic $(68.0 \%)$ and non-diabetic patients (74.3\%). In a multivariable Cox model, ACR $(p<0.0001)$ and diabetes status $(p=0.01)$ were associated with adverse outcome even when several other clinical variables were included in the model. Furthermore, a negative interaction was found between diabetes and ACR $(p=0.01)$. Conclusions/interpretation. Microalbuminuria frequently occurs in diabetic and non-diabetic AMI patients during the first 3 days of admission to hospital and can be used to identify subjects at high risk of mortality.

Keywords Acute myocardial infarction - Albumin : creatinine ratio $\cdot$ Albumin excretion - Diabetes mellitus $\cdot$ Microalbuminuria $\cdot$ Mortality
Received: 11 February 2004 / Accepted: 11 June 2004 Published online: 18 August 2004

C) Springer-Verlag 2004

P. Palatini $(\bullet)$

Department of Clinical and Experimental Medicine,

University of Padova, Clinica Medica 4,

Via Giustiniani, 2, 35128 Padova, Italy

E-mail: palatini@unipd.it

Tel.: +39-049-8212278, Fax: +39-049-8754179

Abbreviations: ACR, albumin : creatinine ratio - AMI, acute myocardial infarction - CK-MB, myocardial band isoenzyme of creatine kinase $\cdot$ LVEF, left ventricular ejection fraction

\section{Introduction}

The prevalence of diabetes mellitus has been rising worldwide due to increasing obesity and decreasing physical activity $[1,2]$. Diabetes affects 6 to $7 \%$ of the Western population $[1,2]$ but is present in as many as $30 \%$ of patients hospitalised with acute coronary syndromes, who also have a worse prognosis than patients without diabetes $[3,4]$. In fact, diabetic subjects experience greater morbidity during the acute phase of myocardial infarction (AMI) and higher mortality in the post-infarction period $[3,5,6]$. This fact holds true even in the era of thrombolytics and primary percutaneous transluminal coronary angioplasty $[7,8]$. 
Low-level urinary albumin has been shown to predict all-cause (largely cardiovascular) mortality in patients with diabetes $[9,10]$. We recently demonstrated that the AER is increased in patients with AMI and it is strongly and independently associated with global and cardiovascular mortality [11, 12].

To our knowledge, no study to date has investigated the time-course and the predictive value of AER for mortality in diabetic patients during AMI. In particular, it is not known whether diabetes status and AER have an additive effect on the prognosis in this clinical setting.

This paper reports on a 3-year prospective study of an unselected sample of diabetic patients with AMI and an age- and sex-matched group of non-diabetic AMI patients. The aims of this study were: (i) to compare the urinary albumin levels in diabetic AMI patients with those in non-diabetic AMI patients and in diabetic control subjects without AMI; and (ii) to investigate whether the presence of microalbuminuria predicts mortality after AMI in diabetic and non-diabetic patients.

\section{Subjects and methods}

Patients. A total of 121 consecutive, unselected diabetic patients (four with Type 1 diabetes and 117 with Type 2 diabetes) and 121 age- and sex-matched non-diabetic patients matched for age and sex were studied. Subjects were admitted to the intensive care units at Bassano del Grappa, Adria and Conegliano General Hospitals (all in northeast Italy) for definite AMI and were prospectively studied. Subjects with urinary tract infections or other concomitant clinical situations that could affect urinary albumin were excluded. In order to compare the baseline AER values, a smaller group of 61 diabetic outpatients (three with Type 1 diabetes and 58 with Type 2 diabetes) who had been admitted to the Diabetic Office of the Internal Medicine Department at Conegliano General Hospital were assessed as control subjects. Having given written informed consent, the patients were interviewed by a physician, who completed a standard record form covering details of past medical history. The study was approved by the hospitals' ethics committees.

A diagnosis of AMI was made if two or more of the following criteria were fulfilled: (i) central chest pain lasting longer than $30 \mathrm{~min}$; (ii) typical changes in serum enzymes (total creatine kinase, myocardial band isoenzyme of creatine kinase [CK$\mathrm{MB}$, aspartate aminotransferase, lactate dehydrogenase); and (iii) typical ECG changes with pathological Q-waves and/or localised ST-T changes in at least two contiguous leads. A patient was defined as having diabetes if there was written documentation that a physician had diagnosed Type 1 diabetes or Type 2 diabetes either before or at the time of the current myocardial infarction. Glycosylated haemoglobin was assayed for participants with diabetes at the local laboratory of each study centre.

Measurements. All clinical and laboratory data were obtained during the first 7 days of hospitalisation. Levels of serum enzymes were measured and a 12-lead ECG was recorded upon admission and every $4 \mathrm{~h}$ thereafter for the first 2 days of admission. These assessments were performed daily as of the 3rd day of admission. Venous blood was drawn for determination of blood glucose and serum levels of total and HDL cholester- ol, triglycerides and creatinine. Blood pressure was measured by specially trained nurses, using a mercury sphygmomanometer with a cuff of appropriate size; the mean of three recordings was used. Heart rate was measured by palpation of the radial pulse. The presence and degree of heart failure was assessed on the 1st, 3rd and 7th days of admission according to Killip classification.

Left ventricular ejection fraction (LVEF) was assessed by two-dimensional echocardiography between the 3rd and 7th day of admission. Four- and two-chamber apical views were recorded on VHS cassettes and sent to Conegliano Hospital, where two physicians with no knowledge of patients' clinical data examined them. LVEF determination was performed according to Simson's method, and the mean of the two measurements was used [13]. Technically unsatisfactory echocardiographic images $(n=35$ among diabetic patients, $n=33$ among non-diabetic patients) were discarded from the analysis.

Urinary albumin excretion. Urinary albumin excretion was assessed in three 24-h urinary collections, which were performed on the 1st, 3rd and 7th days of admission. Urinary collections were made under the supervision of trained nurses to minimise errors in diuresis measurement. Immediately after completion, volumes were measured and urine specimens were frozen $\left(-20{ }^{\circ} \mathrm{C}\right)$ and sent to the University of Padova (Padua, Italy). Here, urinary albumin was measured by RIA using a Human ALB.KIT-double antibody (Techno Genetics, Cassina De Pecchi, Milan, Italy) [14]. The detection limit of the method was $0.5 \mu \mathrm{g} / \mathrm{ml}$ and the between-batch coefficient of variation was $5 \%$. For each 24-h urine sample, creatininuria was also measured using the Jaffe method [15]. Urinary albumin excretion was expressed as the ratio of albumin $(\mu \mathrm{g} / \mathrm{ml})$ : creatinine $(\mathrm{mg} / \mathrm{ml})(\mathrm{ACR}[\mu \mathrm{g} / \mathrm{mg}])$. Microalbuminuria was defined as an ACR of $30 \mu \mathrm{g} / \mathrm{mg}$ or higher for both men and women. All patients enrolled were included in the follow-up. Standard urinalysis was performed at the time of urinary sample collections. Creatinine clearance $(\mathrm{ml} / \mathrm{s})$ was measured on the $1 \mathrm{st}$, 3rd and 7 th days as follows: concentration of creatinine in urine $\times$ urine flow / concentration of creatinine in plasma. Diabetic non-AMI control subjects, who were not hospitalised, were requested to avoid any physical activity (i.e. work and sports activity) and to rest at home during the urine collection period.

Follow-up. For 3 years after recruitment each AMI patient was called for an annual clinical check-up, such that each patient was followed-up for a period of exactly 3 years starting from the day of admission to the intensive care unit. For those who died during a hospital stay, the date and cause of death were obtained from hospital records (including the post-mortem report where available). Mortality data for those who died out of hospital were obtained from the family doctor. No patient dropped out during follow-up. Global mortality and total cardiovascular mortality were used as endpoints in this study. Any instances of coronary artery bypass graft surgery or percutaneous coronary angioplasty performed during the 3 years of follow-up were recorded and used as covariates in the multivariable survival models.

Statistical methods. Statistical analyses were performed using the Systat 7.0 for Windows package (SPSS, Evanston, Ill., USA) and JMP 3.1.4 for Windows (SAS Institute, Cary, N.C., USA). In the matching procedure, age was expressed in days. To correct for positively skewed distributions for ACR, log transformations were used as appropriate. ACR was analysed as a continuous variable, as quartiles and as a dichotomous variable using the cut-off value of $30 \mu \mathrm{g} / \mathrm{mg}$, which is widely used in the literature as a threshold for microalbuminuria. 
Table 1. Baseline characteristics of the patients with acute myocardial infarction

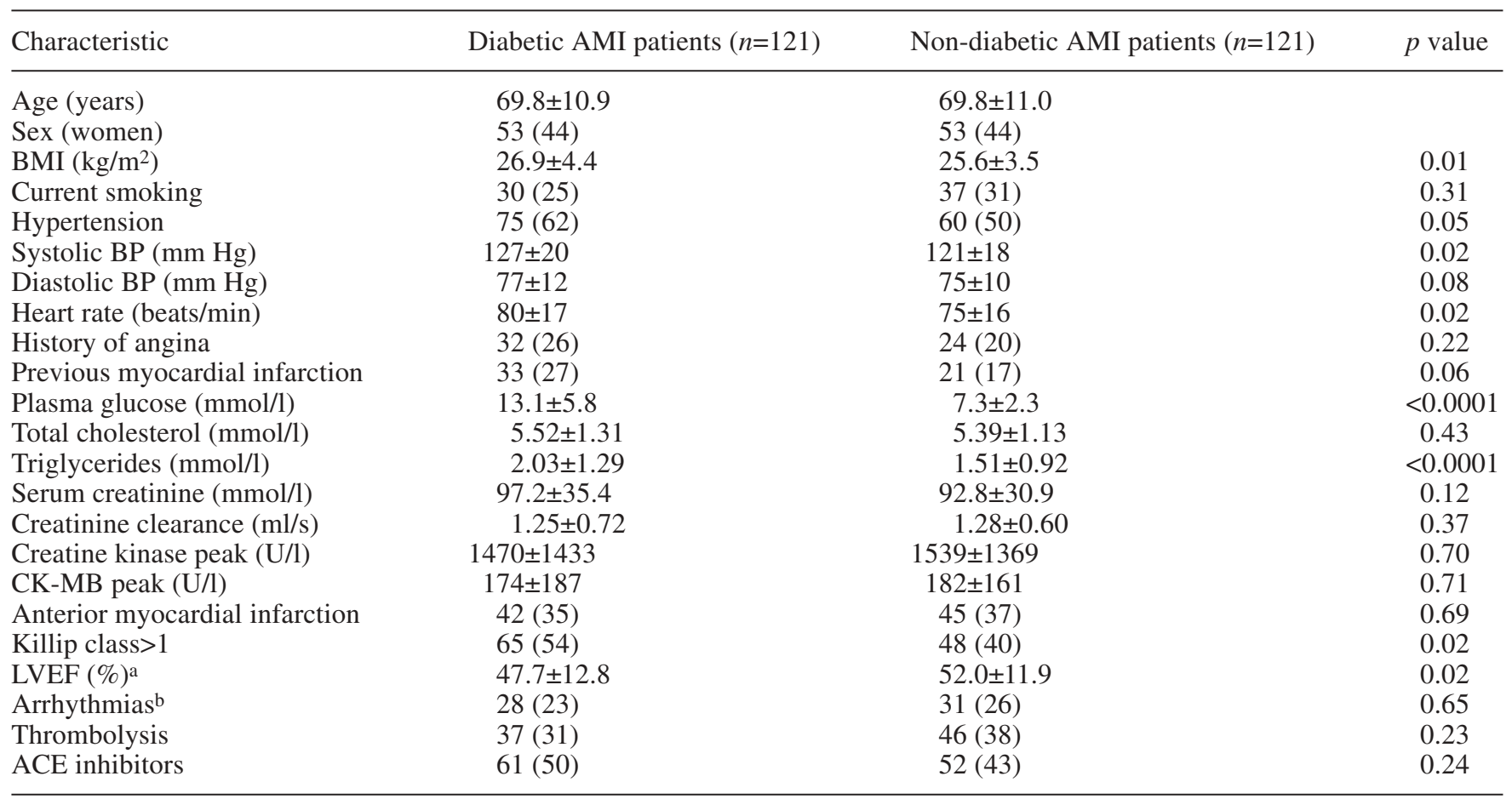

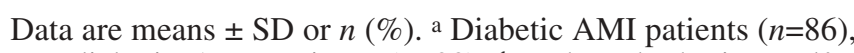
non-diabetic AMI patients $(n=88) ; \mathrm{b}$ tachyarrhythmias and/or bradyarrhythmias during the first week of hospitalisation.
CK-MB, myocardial band isoenzyme of creatine kinase; LVEF, left ventricular ejection fraction
For continuous variables, comparison between groups were made using the unpaired Student's $t$ test and repeated measures analysis of covariance with Tukey tests when data from all 3 days of observation were simultaneously assessed. The covariates used in each comparison are reported in the results section. The chi square test was used for categorical variables. Survival analyses using Cox proportional hazard regression models were completed to assess significant predictors of survival time. All of the risk factors considered significant based on univariable Cox analyses were entered into initial multivariable Cox models. These models were reduced by removing each variable that was non-significant and/or causing the least change in significance. This procedure was continued until no further variables could be removed without producing a significant change in the model [16]. These final models were determined to be the "parsimonious" multivariable models. Survival curves were constructed using the Kaplan-Meier method and compared by the log-rank test. Relative risks were derived from Cox regression models.

Data are presented as means \pm SD for continuous measures, unless otherwise specified and as number of subjects $(\%)$ for categorical variables. All $p$ values are two-tailed and $p$ values less than 0.05 were considered to be statistically significant.

\section{Results}

Patient characteristics. The main clinical characteristics of the diabetic and non-diabetic AMI patients are reported in Table 1. Diabetic AMI patients had higher mean values for BMI, systolic blood pressure, heart rate and triglycerides and a lower mean LVEF than non-diabetic AMI patients. Heart failure (Killip Class $>1$ ) was more frequent among diabetic than nondiabetic AMI patients. The other clinical variables, including treatment with thrombolytics or ACE inhibitors, did not differ significantly between the two groups. Diabetic control outpatients were similar to the diabetic AMI patients with respect to age and sex (age 69.5 \pm 8.8 years, 27 females [44\%]). However, duration of diabetes was longer in the control group $(14.0 \pm 9.5$ vs $9.3 \pm 8.2$ years, $p=0.001)$ and glycosylated haemoglobin level was higher $(7.9 \pm 1.5 \%$ vs $7.0 \pm 1.3 \%$, $p=0.01)$. Systolic BP $(153 \pm 22$ vs $127 \pm 20 \mathrm{~mm} \mathrm{Hg}$, $p<0.0001)$ and diastolic BP $(86 \pm 11$ vs $77 \pm 12 \mathrm{~mm} \mathrm{Hg}$, $p<0.0001)$ were also higher among diabetic outpatients than diabetic AMI patients. Furthermore, serum creatinine was lower in the control group $(61.9 \pm 17.7$ vs $97.2 \pm 35.4 \mathrm{mmol} / 1, p<0.0001)$ and, accordingly, creatinine clearance was higher $(2.14 \pm 0.96$ vs $1.25 \pm$ $0.72 \mathrm{ml} / \mathrm{s}, p<0.0001)$. None of the diabetic outpatients showed clinical signs of heart failure during the week of study. In the diabetic AMI group, 50\% of subjects used insulin and 26\% used oral antidiabetic agents; the corresponding values in the diabetic non-AMI group were $32 \%(p=0.008)$ and $63 \%(p<0.0001)$ respectively.

Albumin : creatinine ratios. Among the diabetic patients, the prevalence of microalbuminuria was much higher in the AMI patients than in the non-AMI 
Table 2. Albumin : creatinine ratio measured on the 1st, 3rd and 7th days of the study

\begin{tabular}{|c|c|c|c|c|c|}
\hline Characteristic & $\begin{array}{l}\text { Diabetic } \\
\text { AMI patients }\end{array}$ & $\begin{array}{l}\text { Non-diabetic } \\
\text { AMI patients }\end{array}$ & $\begin{array}{l}\text { Diabetic } \\
\text { control } \\
\text { subjects }\end{array}$ & $\begin{array}{l}\text { Diabetic } \\
\text { AMI patients vs } \\
\text { non-diabetic } \\
\text { AMI patients, } \\
\text { adjusted } p \text { value }\end{array}$ & $\begin{array}{l}\text { Diabetic } \\
\text { AMI patients vs } \\
\text { diabetic control } \\
\text { subjects, } \\
\text { adjusted } p \text { value }\end{array}$ \\
\hline 1st day ACR $(\mu \mathrm{g} / \mathrm{mg})$ & $122.8 \pm 16.0$ & $91.6 \pm 13.7$ & $27.2 \pm 5.6$ & 0.33 & $<0.0001$ \\
\hline 3rd day ACR $(\mu \mathrm{g} / \mathrm{mg})$ & $52.8 \pm 8.0$ & $36.5 \pm 6.7$ & $20.5 \pm 4.2$ & 0.08 & 0.001 \\
\hline
\end{tabular}

Data are means \pm SEM. a Repeated measures adjusted $F=1.1$, $p=0.28$ vs non-diabetic AMI patients; linear polynomial test for trend $p=0.39$ vs non-diabetic AMI patients; repeated mea-

control outpatients on the 1 st day (62\% vs $28 \%$, $p<0.0001)$ and 3rd day (41\% vs $20 \%, p=0.004)$ of admission. This difference was not significant by the 7 th day $(28 \%$ vs $20 \%, p=0.21)$. ACR was significantly higher in the diabetic subjects with AMI than in the diabetic control group, even after adjusting for blood pressure, creatinine clearance, Killip class and administration of the various drugs (including insulin and oral antidiabetic agents) (Table 2). The Tukey test showed that the between-group differences were significant for all 3 days of measurement. Diabetic AMI patients had high ACR values on the 1st day of admission, which gradually decreased to the 7 th day, whereas the diabetic control subjects had stable ACR values, producing a significant linear difference between the weekly ACR slopes for the two groups (Table 2).

The prevalence of microalbuminuria was lower in the non-diabetic AMI patients than in the diabetic AMI patients $(46 \%, 29 \%$ and $17 \%, p=0.01, p=0.04$ and $p=0.57$ respectively vs diabetic AMI patients). Univariable repeated measures ANOVA revealed that ACR was lower in the non-diabetic AMI patients than in the diabetic AMI patients $(F=4.0, p=0.04)$ but the betweengroup differences were no longer significant after adjusting for BMI, BP and Killip class (Table 2). No significant difference was observed between these two groups with respect to the weekly ACR slopes (Table 2).

Three-year mortality according to diabetes status and albumin : creatinine ratio. During the 3 years of follow-up, 58 patients in the diabetic AMI group (48\%, 24 men and 34 women) and 36 patients in the non-diabetic AMI group (30\%, 15 men and 21 women) died ( $p=0.004)$. Among the diabetic subjects, the causes of death were: reinfarction $(n=7)$, stroke $(n=6)$, heart failure or cardiogenic shock $(n=16)$, sudden death $(n=11)$, other cardiovascular causes $(n=13)$ and non-cardiovascular causes $(n=5)$. Among the non-diabetic patients, causes of death had a similar distribution ( $p=0.26$ vs diabetic patients): reinfarction $(n=2)$, stroke $(n=2)$, heart failure or cardiogenic shock $(n=12)$, sudden death $(n=8)$, other cardiovascular causes $(n=4)$ and non-cardiovascular causes $(n=8)$. sures adjusted $F=18.7, p<0.0001$ vs diabetic control subjects; linear polynomial test for trend $p<0.0001$ vs diabetic control subjects
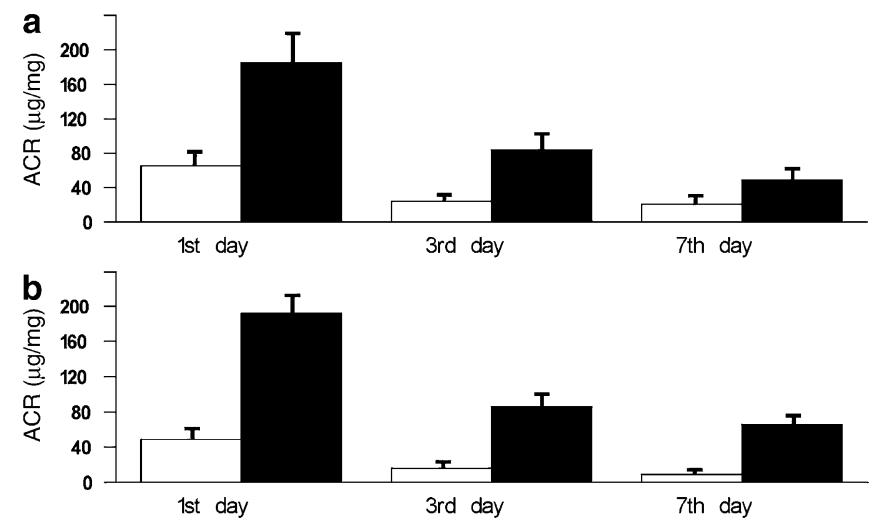

Fig. 1. ACR in diabetic patients (a) and non-diabetic patients (b) with AMI measured on the 1st, 3rd and 7th days of admission to hospital. The graphs of the two groups show patients who died (black bars) and those who survived (open bars). Values are expressed as means \pm SEM. Results of repeated measures analysis of covariance adjusting for CK-MB peak, Killip class, ACE inhibitor and thrombolytic therapy: $F=10.6$, $p=0.001$ in diabetic patients and $F=30.4, p<0.0001$ in non-diabetic patients

Diabetic AMI patients used calcium-channel blockers less frequently than non-diabetic AMI patients $(27 \%$ vs $45 \%, p=0.02)$ during follow-up, while nitrates ( $70 \%$ vs $71 \%, p=0.92$ ), beta-blockers $(30 \%$ vs $34 \%$, $p=0.61)$ and ACE-inhibitors (46\% vs $48 \%, p=0.79$ ) were used by a similar number of patients in the two groups. Compared with the AMI patients who died, survivors were more likely to use beta-blockers $(32 \%$ vs $16 \%, p=0.004)$ and calcium-channel blockers $(37 \%$ vs $19 \%, p=0.003)$, and less likely to use nitrates $(70 \%$ vs $85 \%, p=0.008)$ and insulin (5\% vs $29 \%, p<0.001)$. There was no difference between these two groups with respect to the use of ACE inhibitors (47\% vs $52 \%, p=0.46$ ).

Throughout the first week after admission for AMI, ACR was consistently higher in the patients who died than in those who survived, both among diabetic and non-diabetic individuals (Fig. 1). Secondary analysis showed that the between-group differences were significant for all three measurements $(p<0.001$ for each 
day for diabetic subjects and $p<0.0001$ for each day for non-diabetic subjects). Similar differences were found when deaths from cardiovascular causes alone were considered $(p<0.001$ for diabetic patients and $p<0.0001$ for non-diabetic patients). Figure 2 shows the all-cause mortality rate in diabetic and non-diabetic AMI patients stratified into quartiles according to ACR value on the 3rd day. Both diabetic and non-diabetic subjects exhibited a progressive rise in mortality rate with increasing ACR value $\left(p<0.0001\right.$ for $\chi^{2}$ test for both groups). Mortality rate ranged from $0 \%$ for the nondiabetic patients in the lowest ACR quartile to $73.3 \%$ for the diabetic patients in the highest ACR quartile. Similar results were obtained using measurements taken on the 1st day and the 7th day of admission.

AMI patients were subsequently stratified into four groups according to diabetes status and the absence or presence of microalbuminuria. Figure 3 shows the Kaplan-Meier curves for 3-year mortality for the four groups. The mortality rate was dramatically increased in patients with ACR values greater than or equal to $30 \mu \mathrm{g} / \mathrm{mg}$ on the $3 \mathrm{rd}$ day $(68.0 \%$ among diabetic patients, and $74.3 \%$ among non-diabetic patients). The mortality rate was much lower in patients with ACR values lower than $30 \mu \mathrm{g} / \mathrm{mg}$ on the 3rd day, and was significantly different between patients with and without diabetes (33.8\% vs $11.6 \%$ respectively, $p=0.001$ ). Similar mortality trends were observed for measurements made on the 1st day and the 7th day (log-rank test $p<0.0001$ for both).

Multivariable analyses. Based on separate Cox survival analyses, both diabetes status and ACR were associated with 3-year all-cause mortality (Table 3). Multivariable Cox survival models in which both diabetes status and ACR level were included showed that both of these two predictors were still significantly associated with adverse outcome even after adjustment for seven other clinical variables in the final parsimonious survival model. The predictive power of ACR for mortality remained significant when subjects were

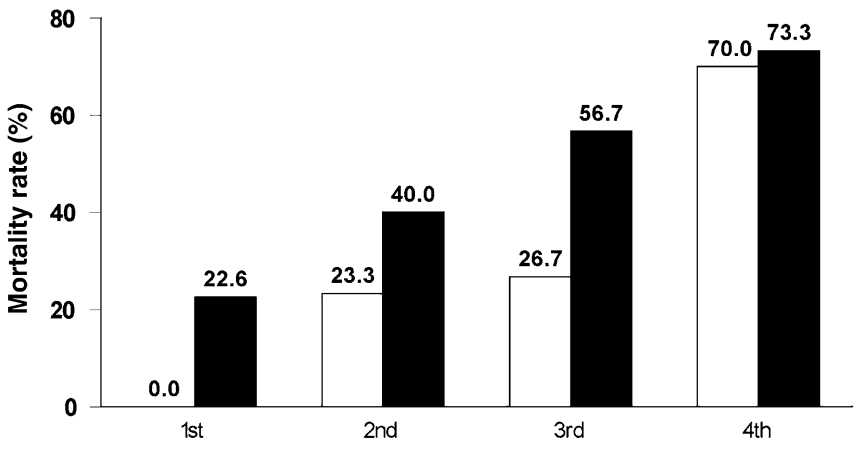

Fig. 2. All-cause mortality rates in diabetic AMI patients (black bars) and non-diabetic AMI patients (open bars) after 3 years of follow-up stratified into quartiles according to ACR values measured on the $3 \mathrm{rd}$ day of admission

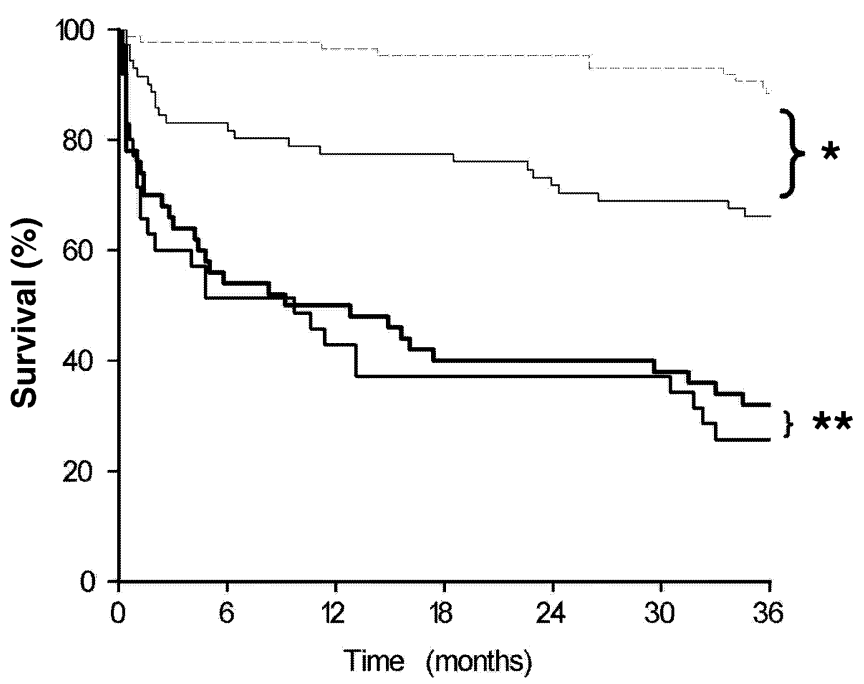

Fig. 3. Kaplan-Meier curves of 3-year all-cause mortality in AMI patients stratified according to diabetes status and the presence of microalbuminuria (ACR $\geq 30 \mu \mathrm{g} / \mathrm{mg}$ ) on the 3 rd day of admission. Log-rank test: $\chi^{2}=79.6, p<0.0001$. * $p=0.001$, $* * p=0.53$. Interrupted line, non-diabetic patients with normoalbuminuria; thin solid line, diabetic patients with normoalbuminuria; medium solid line, non-diabetic patients with microalbuminuria; thick solid line, diabetic patients with microalbuminuria

Table 3. Univariable and multivariable analyses of predictors of 3-year all-cause mortality based on Cox regression models in patients with acute myocardial infarction using data collected on the 3rd day of admission

\begin{tabular}{|c|c|c|c|c|}
\hline Variables & $\mathrm{RR}(95 \% \mathrm{CI})^{\mathrm{a}}$ & $p$ value & $\mathrm{RR}(95 \% \mathrm{CI})^{\mathrm{a}}$ & $p$ value \\
\hline ACR & $2.8(2.2-3.7)$ & $<0.0001$ & $2.2(1.7-2.9)$ & $<0.0001$ \\
\hline Thrombolysis & $0.3(0.2-0.6)$ & $<0.0001$ & $0.2(0.1-0.5)$ & $<0.0001$ \\
\hline Systolic BP & $0.8(0.7-1.0)$ & 0.14 & $0.7(0.5-0.9)$ & 0.006 \\
\hline Age & $2.2(1.7-2.8)$ & $<0.0001$ & $1.6(1.2-2.1)$ & 0.0009 \\
\hline LVEF ( $n=174$ of 242$)$ & $0.5(0.4-0.6)$ & $<0.0001$ & $0.7(0.5-0.9)$ & 0.01 \\
\hline Killip class $(1-4)$ & $2.5(2.0-3.1)$ & $<0.0001$ & $1.5(1.1-2.0)$ & 0.01 \\
\hline Interaction between diabetes and ACR & $0.4(0.2-0.8)$ & 0.003 & $0.5(0.3-0.8)$ & 0.01 \\
\hline
\end{tabular}

Units of variables as reported in Table 1. a Increased risk of mortality for a 1-SD increase in continuous variables. CK-MB, myocardial band isoenzyme of creatine kinase; LVEF, left ventricular ejection fraction; RR, relative risk 
divided according to age ( $<65$ years: $n=74, \chi^{2}=11.9$, $p=0.0006$; $\geq 65$ years: $\left.n=168, \chi^{2}=22.6, p<0.0001\right)$. When ACR was excluded from the model, the predictive power of diabetes status for mortality increased $\left(\chi^{2}=10.2, p=0.001\right)$. It is noteworthy that ACR was by far the strongest predictor of all the variables considered. Furthermore, a negative interaction was found between diabetes and ACR, both at a univariable and a multivariable level (Table 3). Thrombolytic therapy favourably affected outcome. Sex, BMI, current smoking, pre-existing hypertension, prior myocardial infarction and anterior site of AMI were all significant predictors of mortality based on univariable tests, but they did not reach statistical significance when entered into multivariable models. The same multivariable analyses performed using data from the 1st and 7 th days of admission produced similar results (1st day: diabetes status: $\chi^{2}=6.0, p=0.01$; ACR: $\chi^{2}=24.3$, $p<0.0001$; 7 th day: diabetes status: $\chi^{2}=5.2, p=0.01$; ACR: $\left.\chi^{2}=21.5, p<0.0001\right)$. Similar associations were found for cardiovascular mortality.

\section{Discussion}

The results of the present study indicate that ACR is dramatically increased during the first 3 days after AMI in diabetic patients, and that during this period microalbuminuria is more common in diabetic than in non-diabetic individuals. Although we cannot provide direct evidence that the increase in ACR is due to AMI (as no ACR values prior to AMI are available), the marked difference in ACR between diabetic AMI patients and diabetic non-AMI outpatients on the $1 \mathrm{st}$ and 3rd days of admission support this concept. Furthermore, microalbuminuria strongly predicts cardiovascular and all-cause mortality in the setting of AMI. The association between microalbuminuria and the risk of mortality was independent of other cardiovascular risk factors. When the two groups of AMI patients were stratified into quartiles according to ACR values, the mortality rate ranged from $0 \%$ for the nondiabetic AMI patients in the lowest ACR quartile to $73.3 \%$ for the diabetic AMI patients in the highest ACR quartile. Our group has previously reported that low-level urinary albumin predicts in-hospital and 1-year mortality in subjects admitted to hospital for AMI [11, 12]. The present study is the first to examine the additional prognostic information provided by albuminuria for 3-year mortality over that already available from clinical and instrumental findings in diabetic patients with AMI. The present results are consistent with those recently observed in a population of hypertensive subjects with left ventricular hypertrophy who were enrolled in the LIFE study [17]. In this study, Wachtell and colleagues demonstrated a strong association between ACR and the risk of cardiovascular mortality in both diabetic and non-diabetic individuals.
A number of studies performed before the introduction of fibrinolysis for the treatment of patients with AMI consistently showed a higher mortality rate in diabetic patients $[3,5,6]$. Although current treatment of AMI with fibrinolytic agents and aspirin has led to a marked improvement in the prognosis for AMI [18, 19], diabetic patients remain a subgroup at high risk of mortality, both in hospital and after discharge, and diabetes still doubles the case fatality rate $[5,7,20]$. The results of the present study confirm this, as diabetes status in itself accounted for an increase in the risk of 3 -year global mortality by a factor of 1.9. The risk was 1.2 times higher in the diabetic subjects without microalbuminuria, but 3.5 times higher in the subjects with ACR values above or equal to $30 \mu \mathrm{g} / \mathrm{ml}$.

The increased mortality in diabetic patients may be accounted for by several mechanisms, and it is in this context that new information on this topic must be evaluated. Among factors such as more extensive coronary atherosclerosis in diabetic subjects, alterations in the fibrinolytic system that facilitate reocclusion after fibrinolysis [21, 22], diabetic cardiomyopathy with systolic or diastolic dysfunction [23], and autonomic imbalance [24], particular emphasis has been placed on endothelial dysfunction leading to impaired myocardial perfusion [25]. Albuminuria is considered to be a marker of widespread endothelial dysfunction enhancing atherogenesis, and several studies have shown that the mortality risk is 2.3 to 4 times higher in diabetic patients with microalbuminuria than in those without $[26,27,28]$. Thus, it is possible that the glomerular albumin leak reflects a widespread atherosclerosis-mediated capillary vasculopathy $[25,26,27$, 29]. Although we have no ACR values for the patients prior to AMI, it is reasonable to assume that the large difference in ACR between the diabetic AMI group and the diabetic control group is accounted for by AMI. Therefore, it is conceivable that the inflammatory injury and haemodynamic changes related to AMI [12] caused a greater leakage of urinary albumin in subjects with diabetes and associated glomerular damage. A recent study has demonstrated an association between C-reactive protein (a sensitive marker of inflammation) and microalbuminuria [30], and previous results from our laboratory indicate that $\mathrm{C}$-reactive protein is a strong independent predictor of mortality in subjects with AMI [31]. The negative interaction between diabetes and ACR with respect to mortality suggests that microalbuminuria is the overriding factor in the setting of AMI because it reflects a heavily compromised clinical situation, and microalbuminuria was more common among the diabetic individuals than the non-diabetic individuals. In contrast, within the AMI patients with normal ACR, diabetes still retained an independent predictive power for mortality.

Albuminuria has been associated with adverse changes in several cardiovascular risk factors [27, 29]. A critical question within this context is whether the 
relationship between increased ACR and mortality merely reflects the association between albuminuria and other predictors of mortality. However, in our analyses, ACR remained a strong predictor of mortality even after adjustment for these factors, suggesting that ACR is an independent additive component in the relationship between albuminuria and mortality in patients with AMI. A possible limitation of this study is that the majority of enrolled patients were elderly subjects, and thus the present results may not apply to younger individuals. However, the predictive power of ACR for mortality was also present in subjects younger than 65 years of age.

In conclusion, the present data show that microalbuminuria is common in diabetic AMI patients and provides prognostic information additional to that provided by other well-known indicators of risk. This relatively inexpensive and widely available test can help to identify subjects at high risk of mortality, for whom additional preventive and therapeutic measures are advisable.

Acknowledgements. We thank S. Pianca, S. Petucco, P. Michelazzo and J. Civiero for their help in data handling. We would also like to thank the nurses at the emergency care units for patient management. There were no conflicts of interest in this study.

\section{References}

1. Grundy SM, Howard B, Smith S, Eckel R, Redberg R, Bonow RO (2002) Prevention conference VI: diabetes and cardiovascular disease: executive summary. Circulation 105:2231-2239

2. DECODE Study Group (2003) Age- and sex-specific prevalences of diabetes and impaired glucose regulation in 13 European cohorts. Diabetes Care 26:61-69

3. Abbud ZA, Shindler DM, Wilson AC, Kostis JB (1995) Effect of diabetes on short- and long-term mortality rates of patients with acute myocardial infarction: a state-wide study. Myocardial Infarction Data Acquisition System Study Group. Am Heart J 130:51-58

4. Carson JL, Scholz PM, Chen AY, Peterson ED, Gold J, Schneider SH (2002) Diabetes mellitus increases shortterm mortality and morbidity in patients undergoing coronary artery bypass graft surgery. J Am Coll Cardiol 40:418-423

5. Karlson BW, Herlitz J, Hjalmarson A (1993) Prognosis of acute myocardial infarction in diabetic and non-diabetic patients. Diabet Med 10:449-454

6. Melchior T, Kober L, Madsen CR et al. (1999) Accelerating impact of diabetes mellitus on mortality in the years following an acute myocardial infarction. TRACE Study Group. Trandolapril Cardiac Evaluation. Eur Heart J 20:973-978

7. Mak KH, Moliterno DJ, Granger CB et al. (1997) Influence of diabetes mellitus on clinical outcome in the thrombolytic era of acute myocardial infarction. GUSTO-I Investigators. Global Utilization of Streptokinase and Tissue Plasminogen Activator for Occluded Coronary Arteries. J Am Coll Cardiol 30:171-179
8. Hsu LF, Mak KH, Lau KW et al. (2002) Clinical outcomes of patients with diabetes mellitus and acute myocardial infarction treated with primary angioplasty or fibrinolysis. Heart 88:260-265

9. Hillege HL, Fidler V, Diercks GF et al. (2002) Urinary albumin excretion predicts cardiovascular and noncardiovascular mortality in general population. Circulation 106:1777-1782

10. Gerstein HC, Mann JF, Yi Q et al. (2001) Albuminuria and risk of cardiovascular events, death, and heart failure in diabetic and nondiabetic individuals. HOPE Study Investigators. JAMA 286:421-426

11. Berton G, Citro T, Palmieri R, Petucco S, De Toni R, Palatini P (1997) Albumin excretion rate increases during acute myocardial infarction and strongly predicts early mortality. Circulation 96:3338-3345

12. Berton G, Cordiano R, Palmieri R, Cucchini F, De Toni R, Palatini P (2001) Microalbuminuria during acute myocardial infarction: a strong predictor for one-year mortality. Eur Heart J 22:1466-1475

13. Erbel R (1982) Comparison of single-plane and biplane volume determination by two-dimensional echocardiography: 1. Asymmetric model hearts. Eur Heart J 3:469-474

14. Bradows RG, Nichols D, Shaker G, Kubasik NP (1986) Evaluation of a new radioimmunoassay for urinary albumin. Diabetes Care 9:189-193

15. Jensen JS, Clausen P, Borch-Johnsen K, Jensen G, FeldtRasmussen B (1997) Detecting microalbuminuria by urinary albumin/creatinine concentration ratio. Nephrol Dial Transplant 12 [Suppl 2]:6-9

16. Altman DG (1992) Modelling survival, the Cox regression model. In: Practical statistics for medical research. Chapman \& Hall, London, pp 387-394

17. Wachtell K, Ibsen H, Olsen MH et al. (2003) Albuminuria and cardiovascular risk in hypertensive patients with left ventricular hypertrophy: the LIFE study. Ann Intern Med 139:901-906

18. Woodfield SL, Lundergan CF, Reiner JS et al. (1996) Angiographic findings and outcome in diabetic patients treated with thrombolytic therapy for acute myocardial infarction: the GUSTO-I experience. J Am Coll Cardiol 28:1661-1669

19. Mak KH, Moliterno DJ, Granger CB et al. (1997) Influence of diabetes mellitus on clinical outcome in the thrombolytic era of acute myocardial infarction. J Am Coll Cardiol 30:171-179

20. Zuanetti G, Latini R, Maggioni AP, Santoro L, Franzosi MG (1993) Influence of diabetes on mortality in acute myocardial infarction: data from the GISSI-2 study. J Am Coll Cardiol 22:1788-1794

21. McGill JB, Schneider DJ, Arfken CL, Lucore CL, Sobel BE (1994) Factors responsible for impaired fibrinolysis in obese subjects and NIDDM patients. Diabetes 43:104-109

22. Piemontino U, Ceriello A, di Minno G (1994) Hemostatic and metabolic abnormalities in diabetes mellitus. The search for a link. Haematologica 79:387-392

23. Standl E, Schnell O (2000) A new look at the heart in diabetes mellitus: from ailing to failing. Diabetologia 43:1455-1469

24. King SB 3rd (2000) Acute myocardial infarction: are diabetics different? J Am Coll Cardiol 35:1513-1515

25. Stehouwer CDA, Nauta JJP, Seldenrust GC, Hackeng WHL, Donker AJM, Ottolander GJH den (1992) Urinary albumin excretion, cardiovascular disease, and endothelial dysfunction in non-insulin-dependent diabetes mellitus. Lancet 340:319-323 
26. Garg JP, Bakris GL (2002) Microalbuminuria: marker of vascular dysfunction, risk factor for cardiovascular disease. Vasc Med 7:35-43

27. Pedrinelli R, Dell'Omo G, Di Bello V, Pontremoli R, Mariani M (2002) Microalbuminuria, an integrated marker of cardiovascular risk in essential hypertension. J Hum Hypertens 16:79-89

28. Nelson RG, Pettitt DJ, Carraher MJ, Baird HR, Knowler WC (1988) Effect of proteinuria on mortality in NIDDM. Diabetes 37:1499-1504
29. Halimi JM, Forhan A, Balkau B et al., D.E.S.I.R. Study Group (2001) Is microalbuminuria an integrated risk marker for cardiovascular disease and insulin resistance in both men and women? J Cardiovasc Risk 8:139-146

30. Stuveling EM, Bakker SJ, Hillege HL et al. (2004) C-reactive protein modifies the relationship between blood pressure and microalbuminuria. Hypertension 43:791-796

31. Berton G, Cordiano R, Palmieri R, Pianca S, Pagliara V, Palatini P (2003) C-reactive protein in acute myocardial infarction: Association with heart failure. Am Heart J 145:1094-1101 